\title{
Doxorubicin Binds to Un-phosphorylated Form of hNopp140 and Reduces Protein Kinase CK2-Dependent Phosphorylation of hNopp140
}

\author{
Yun-Kyoung Kim ${ }^{1,2}$, Won Kyu Lee ${ }^{1}$, Youngnam Jin ${ }^{1}$, Kong-Joo Lee ${ }^{2}$, Hyesung Jeon ${ }^{1}$ and Yeon Gyu Yü,* \\ ${ }^{1}$ Biomedical Research Center, Korea Institute of Science and Technology, 39-1, Hwawolkok-dong, Songbuk-gu, Seoul 136-791, Korea \\ ${ }^{2}$ Division of Molecular Life Sciences, Ewha Womans University, Seoul 120-750, Korea \\ ${ }^{3}$ Department of Chemistry, Kookmin University, 861-1, Jeoungneung-dong, Songbuk-gu, Seoul 136-702, Korea
}

Received 12 May 2006, Accepted 11 August 2006

\begin{abstract}
Human nucleolar phosphoprotein p140 (hNopp140) is a nucleolar phosphoprotein that can bind to doxorubicin, an anti-cancer agent. We have examined the interaction between hNopp140 and doxorubicin as well as the folding property of hNopp140. Also, the effects of ATP and phosphorylation on the affinity of hNopp140 to doxorubicin are investigated by affinity dependent co-precipitation and surface plasmon resonance methods. Doxorubicin preferentially binds to un-phosphorylated form of hNopp140 with a $K_{D}$ value of $3.3 \times 10^{-7} \mathrm{M}$. Furthermore, doxorubicin reduces the protein kinase CK2-dependent phosphorylation of hNopp140, indicating that doxorubicin may perturb the cellular function of hNopp140 by reducing the protein kinase CK2-dependent phosphorylation of hNopp140. Low contents of the secondary structures of hNopp140 and the fast rate of proteolysis imply that hNopp140 has a high percentage of flexible regions or extended loop structures.
\end{abstract}

Keywords: Doxorubicin, hNopp140, Phosphorylation, Protein kinase CK2

\section{Introduction}

The nucleolus is a subnuclear compartment in which the transcription of rDNA and the assembly of preribosome particles take place. Proteins have been identified that are associated with the nucleolus and involved in its formation (Olson and Busch, 1978). Human nucleolar phosphoprotein p140 (hNopp140) was identified originally as a shuttle protein between the cytoplasm and nucleolus (Meier and Blobel, 1992). Subsequently, it was shown to interact with the

\footnotetext{
* To whom correspondence should be addressed.

Tel: 82-2-910-4619; Fax: 82-2-910-4415

E-mail: ygyu@kookmin.ac.kr
}

following: RNA polymerase I, which is responsible for the expression of rRNA genes (Yang et al., 2000); p80 coilin (Isaac et al., 1998) and NAP57 (Meier and Blobel, 1994), which are constituents of the coiled body; snRNPs (Yang et al., 2000), and protein kinase CK2 (Li et al., 1997). Specific interactions between hNopp140 and these proteins indicate that hNopp140 plays important roles in the formation of the nucleolus during cell division.

hNopp140 contains multiple phosphorylation sites and is one of the most highly-phosphorylated protein in the cell (Meier and Blobel, 1992). SDS-PAGE analysis identified endogenous hNopp1 40 as a $140 \mathrm{kDa}$ protein rather than the calculated $77 \mathrm{kDa}$ (Pai et al., 1995). Interestingly, the degree of phosphorylation changes over the course of the cell cycle. Although the level of hNopp140 in mitosis is much lower than in interphase, the hNopp140 remaining in mitotic cells is highly phosphorylated (Pai et al., 1996). To date the protein kinases that could phosphorylate hNopp140 were known as casein kinase 2 (protein kinase CK2), cell cycle dependent kinase 2 (cdc2 kinase), and protein kinase A (PKA). Specially, hNopp140 contains 82 protein kinase CK2 target sequences, and it is heavily phosphorylated by this enzyme (Pai et al., 1995). The fluctuations in the expression level of hNopp140 and its phosphorylation state during cell cycle implicates that hNopp140 may have a role in cell cycle. In addition, overexpression of hNopp140 results in altered nucleoli structure, mislocalization of nucleolus proteins, inhibition of rRNA gene transcription, and improper formation of the nucleolus (Chen et al., 1999; Isaac et al., 2000). These observations suggest that hNopp140 is crucial for normal cell growth. The primary structure of hNopp140 is characterized by the presence of repeating charged and hydrophilic regions, and lacks distinct structural or functional motifs. Although the structural properties of hNopp140 have not been studied extensively, those studied to date, along with the presence of a low percentage of nonpolar amino acids, suggest that hNopp140 may be a disordered or unfolded protein in its native state (Fink, 2005). 
Following screening with a T7 phage display human cDNA library, hNopp140 was found to bind immobilized doxorubicin (Jin et al., 2002). Doxorubicin is an antineoplastic agent isolated from Streptomyces peucetius and is used in the treatment of acute leukemia and a wide variety of solid tumors (Blum and Carter, 1974). One of the suggested mechanisms for the cytotoxic activity of doxorubicin is its ability to intercalate with DNA base pairs (Simpkins et al., 1984). The T7 phage displaying the C-terminal half of hNopp140, as well as the thioredoxin fusion protein of its $\mathrm{C}$-terminal region showed strong binding affinity to doxorubicin (Jin et al., 2002). The finding that doxorubicin exhibits a higher affinity for the C-terminus of hNopp140 than DNA suggests that doxorubicin may interact with hNopp140 in vivo and affect its cellular function. However, the detailed interaction between hNopp140 and doxorubicin and the molecular function of hNopp140 remain to be determined.

In this study, we prepared a full-length recombinant hNopp140 and examined its interaction with doxorubicin. The effects of phosphorylation or ATP on the binding affinity of hNopp140 to doxorubicin were investigated. Furthermore, we observed that the phosphorylation level of hNopp140 by protein kinase CK2 was significantly reduced by doxorubicin. Besides, a low percentage of secondary structure was measured in hNopp140 by circular dichroism analysis. These observations indicate that hNopp140 is a putative unfolded protein, and doxorubicin may perturb the phosphorylation state of hNopp140.

\section{Materials and Methods}

Materials. Doxorubicin hydrochloride and streptavidin agarose beads were purchased from Sigma-Aldrich. The CM5 sensor chip and amine coupling kit were acquired from Biacore $\mathrm{AB}$. Biotinylation of doxorubicin have been prepared as described previously (Jin et al., 2002). Protein kinase CK2, PKA and cdc2 kinase were purchased from New England Biolabs. Polyclonal antibodies of hNopp140 were prepared from mouse using purified hNopp 140 . All other chemicals were of reagent grade.

Protein Purification: DNA encoding full-length hNopp140, with an N-terminal His-tag, was cloned into pET28a (Novagen) to form an expression vector of hNopp140. Recombinant hNopp140 was expressed in E. coli BL21(DE3) and purified as previously described (Kim et al., 2003). Following SP-sepharose chromatography, recombinant un-phosphorylated hNopp 140 was purified further by His-tag affinity chromatography using a Ni-NTA column (Invitrogen).

Protein phosphorylation. The protein kinase CK2-mediated protein phosphorylation reaction was performed by incubation of $100 \mathrm{mg}$ substrate proteins with $15 \mathrm{U}$ of protein kinase CK2 in $100 \mu \mathrm{L}$ of reaction buffer (20 mM Tris- $\mathrm{HCl}, 50 \mathrm{mM} \mathrm{KCl}, 10 \mathrm{mM}$ $\mathrm{MgCl}_{2,} 2.5 \mathrm{mM}$ ATP, and $1 \mu \mathrm{M}\left[\gamma_{-}{ }^{32} \mathrm{P}\right]$ ATP $\left(200 \mathrm{cpm}\right.$ pmole $\left.{ }^{-1}\right), \mathrm{pH}$ 7.5) at $30^{\circ} \mathrm{C}$ for $20 \mathrm{~min}$. Prolonged incubation at $30^{\circ} \mathrm{C}$ for $12 \mathrm{hr}$ was used for the preparation of extensively phosphorylated hNopp 140 (phospho-hNopp140). PKA-mediated phosphorylation reactions were performed by incubation of substrate protein with $2.5 \mathrm{U}$ of PKA in $20 \mu \mathrm{L}$ of reaction buffer $(50 \mathrm{mM}$ Tris- $\mathrm{HCl}, 10 \mathrm{mM} \mathrm{MgCl}$, and $200 \mu \mathrm{M}$ ATP, $\mathrm{pH} 7.5)$ containing $1 \mu \mathrm{M}\left[\gamma^{-32} \mathrm{P}\right]$ ATP $(200 \mathrm{cpm}$ pmole $^{-1}$ ) at $30^{\circ} \mathrm{C}$ for $30 \mathrm{~min}$. The reaction with cdc2 kinase was carried out using $3 \mu \mathrm{g}$ substrate protein in $20 \mu \mathrm{L}$ of reaction buffer (50 mM Tris-HCl, $10 \mathrm{mM} \mathrm{MgCl}_{2}, 1 \mathrm{mM}$ EGTA, $2 \mathrm{mM}$ DTT, 0.01\% Brij35, and $300 \mu \mathrm{M}$ ATP, $\mathrm{pH} 7.4)$ containing1 $\mu \mathrm{M}\left[\gamma_{-}{ }^{32} \mathrm{P}\right]$ ATP $\left(200 \mathrm{cpm} \mathrm{pmole}^{-1}\right)$ for $30 \mathrm{~min}$ at $30^{\circ} \mathrm{C}$. The phosphorylation reaction was terminated by heat treatment at $90^{\circ} \mathrm{C}$ for $3 \mathrm{~min}$. The phosphorylation level of hNopp140 was estimated either by monitoring the change in mobility on a $12 \%$ SDS-PAGE or by autoradiograph. Quantification of the protein bands detected by the autoradiography was performed using a FUJIX BAS 2000 Phosphoimager (Fuji).

Pull-down assay of hNopp140 with biotinylated doxorubicin. Un-phosphorylated or phospho-hNopp140 $(2 \mu \mathrm{g})$ were incubated at $4^{\circ} \mathrm{C}$ for $2 \mathrm{~h}$ with $2.5 \mu \mathrm{M}$ biotinylated doxorubicin in binding buffer (10 mM Mops, pH 7.5, $100 \mathrm{mM} \mathrm{NaCl}, 50 \mathrm{mM} \mathrm{KCl}$, and $5 \mathrm{mM}$ $\mathrm{MgCl}_{2}$ ), and mixed with $20 \mu \mathrm{L}$ streptavidin-agarose (50\%) (Sigma) for $2 \mathrm{~h}$ at $4^{\circ} \mathrm{C}$. Then, the agarose beads were recovered by centrifugation, and washed with the binding buffer for 5 times. The proteins bound to the agarose beads were extracted in the SDSPAGE sample buffer, and separated by $12 \%$ SDS-PAGE. For immunoblotting, the proteins the SDS-gel were transferred onto a polyvinylidene difluoride membrane. The membrane was blocked with $5 \%$ non-fat dried milk for $1 \mathrm{~h}$, reacted with $0.2 \mu \mathrm{g} / \mathrm{mL}$ mouse anti-hNopp140 antibodies in PBST (50 mM potassium phosphate, $100 \mathrm{mM} \mathrm{NaCl}, 0.1 \%$ Tween-20, $\mathrm{pH}$ 7.4) for $1 \mathrm{~h}$, then incubated with $0.1 \mu \mathrm{g} / \mathrm{mL}$ sheep anti-mouse IgG conjugated to horseradish peroxidase for $1 \mathrm{~h}$. After successive washes with PBST, immunoblots were visualized using an enhanced chemiluminescence system (Pierce) as described in the instruction manual.

Surface plasmon resonance (SPR) analysis. SPR analysis of hNopp140 binding to doxorubicin was carried out using a BIAcore 3000 (BIAcore AB). Doxorubicin was immobilized on the CM-5 sensor chip, according to the manufacturer's instructions. The surface matrix was activated by a $10 \mathrm{~min}$ injection of $0.2 \mathrm{M} \mathrm{N}$ ethyl-N'-(3-diethylamino-propyl)-carbodiimide and $50 \mathrm{mM} \mathrm{N}$ hydroxysuccinimide. Doxorubicin $(500 \mathrm{mM})$ was then injected in $30 \mathrm{~mL}$ of $10 \mathrm{mM}$ sodium phosphate (pH 7.0), followed by $150 \mathrm{mM}$ $\mathrm{NaCl}$. Remaining N-hydroxysuccinimide reactive groups were inactivated by injection of $1.0 \mathrm{M}$ ethanolamine- $\mathrm{HCl}(\mathrm{pH} 8.5)$ for $10 \mathrm{~min}$. All analyses were performed at a flow rate of $30 \mathrm{~mL} \mathrm{~min}^{-1}$. Prior to loading of analyte, the chip was equilibrated with running buffer (10 mM Mops, $150 \mathrm{mM} \mathrm{NaCl,} \mathrm{1mM} \mathrm{EDTA,} \mathrm{and} \mathrm{pH}$ 7.5). Various concentrations of each protein were prepared in the running buffer and injected over the immobilized doxorubicin. Following injection of the analyte, running buffer was introduced onto the sensor surface to start dissociation. The chip was regenerated by injection of $10 \mathrm{~mL} 0.017 \% \mathrm{SDS}, 30 \mathrm{mM} \mathrm{NaCl}$, and $1.7 \mathrm{mM}$ $\mathrm{NaOH}$. All binding data were analyzed using the BIAevaluation 3.1 software (BIAcore AB).

Cell culture and transfection. The cDNA encoding hNopp140, flanked by $B a m H I$ and $S a l$ I restriction sites, was cloned into the mammalian expression vector pCMV-Tag3B (Stratagene) to 
generate pCMV-hNopp140, which expresses an N-terminal Myctagged hNopp140 fusion protein. 293T cells were grown in $10 \mathrm{~cm}-$ diameter Petri-dishes and maintained in Dulbecco's modified Eagle's medium supplemented with $10 \%(\mathrm{v} / \mathrm{v})$ fetal bovine serum. Subconfluent cells were transiently transfected with pCMVhNopp140 DNA ( $4 \mu \mathrm{g}$ per dish) mixed with LipofectAMINE and PLUS reagent (Invitrogen), according to the manufacturer's protocol. Cells were harvested approximately $48 \mathrm{~h}$ after transfection.

Circular dichroism spectroscopy. Circular dichroism of $50 \mu \mathrm{g} / \mathrm{ml}$ hNopp140 in $50 \mathrm{mM}$ Tris- $\mathrm{HCl}$ and $100 \mathrm{mM} \mathrm{NaCl}, \mathrm{pH} 7.5$ was measured using a Jasco J-715 spectropolarimeter (Jasco) at $25^{\circ} \mathrm{C}$. The secondary structure content was evaluated by computer software J700 (Jasco).

Protease treatment. Proteolysis of hNopp140 was performed in $20 \mathrm{mM}$ Na-phosphate, $300 \mathrm{mM} \mathrm{NaCl}, \mathrm{pH} 7.4$ using subtilisin or protease $\mathrm{K}$. About $0.1 \mathrm{mg} \mathrm{ml}^{-1}$ of hNopp140 was incubated with different concentrations of proteases at $25^{\circ} \mathrm{C}$ for $30 \mathrm{~min}$. Reaction were terminated by addition of SDS-sample buffer and boiling for $5 \mathrm{~min}$. Then, the reaction products were analyzed using SDSPAGE.

\section{Results}

Interaction between hNopp140 and doxorubicin depends on the phosphorylation state and ATP. Previously, we identified the C-terminal domain of hNopp140 as a doxorubicin-binding protein, using a phage display cloning method (Jin et al., 2002). It was also known that hNopp140 was extensively phosphorylated by protein kinase CK2 (Meier and Blobel, 1992) and had ATP/GTPase activities (Chen and Yeh, 1997). To examine the effect of phosphorylation and ATP on the binding affinity of hNopp140 to doxorubicin, a recombinant hNopp140 was prepared and examined its interaction with doxorubicin. Phospho-hNopp140 was prepared from the recombinant hNopp140 after prolonged incubation with protein kinase CK2. The slower migration of phosphohNopp140 in SDS-PAGE indicated an extensive phosphorylation of hNopp140 (Fig. 1A). When the un-phosphorylated or phospho-hNopp140 were incubated with biotinylated doxorubicin and recovered using streptavidin-coated agarose beads, only the un-phosphorylated form was recovered; phospho-hNopp140 failed to bind doxorubicin (Fig. 1B). The effect of ATP on the interaction between hNopp140 and doxorubicin was further examined using SPR analysis. When hNopp140 was eluted on to a sensor chip containing immobilized doxorubicin, it produced an association sensorgram with an association rate constant of $2.6 \times 10^{4} \mathrm{M}^{-1} \mathrm{sec}^{-1}$, and a dissociation sensorgram with a dissociation rate constant of $8.7 \times 10^{-3} \mathrm{sec}^{-1}$ (Fig. 2A). From the concentration-dependent association and dissociation sensorgrams, the apparent binding constant $\left(\mathrm{K}_{\mathrm{D}}\right)$ of hNopp140 to doxorubicin was calculated as $3.3 \times 10^{-7} \mathrm{M}$, which is about 10 fold higher affinity than that of the Cterminal region of hNop1p40 (Jin et al., 2002). The effect of

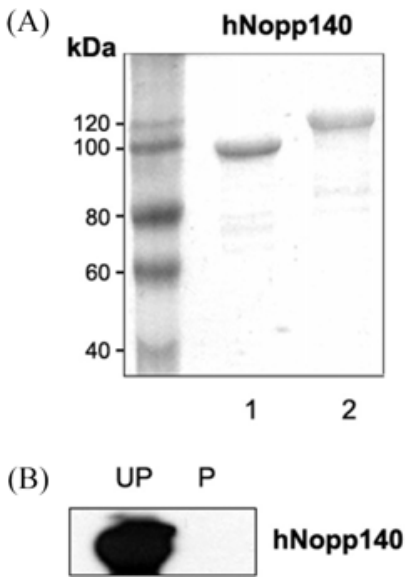

Fig. 1. Phosphorylation of hNopp 140 by protein kinase CK2 reduced its binding affinity to doxorubicin. (A) Recombinant hNopp 140 was incubated with protein kinase CK2 at $30^{\circ} \mathrm{C}$ for $16 \mathrm{~h}$. Un-phosphorylated (lane 1) and phospho-hNopp140 (lane 2) was analyzed by $12 \%$ SDS-PAGE. (B) Unphosphorylated (UP) or phospho-hNopp140 (P) were incubated with biotinylated doxorubicin and streptavidin coated agarose beads. The beads were recovered by centrifugation, and the proteins bound to the beads were eluted and examined by western analysis using antihNopp140 antibodies.

ATP in the range of cellular concentration (Koop and Gabbold, 1993) on the interaction between hNopp140 and doxorubicin was examined using SPR analysis. The binding sensorgram of hNopp140 to doxorubicin was enhanced significantly as the concentration of ATP increased (Fig. 2B). In the presence of $5 \mathrm{mM}$ ATP, the affinity of hNopp140 for doxorubicin increased 10-fold, and the $\mathrm{K}_{\mathrm{D}}$ value was measured as $2.8 \times 10^{-8} \mathrm{M}$. These results indicate that phosphorylation or ATP severely alters the binding affinity of hNopp140 to doxorubicin.

Doxorubicin reduces protein kinase CK2-dependnent phosphorylation of hNopp140. The specific interaction between doxorubicin and hNopp140 suggests that doxorubicin may change the phosphorylation of hNopp140. Since various kinases such as protein kinase CK2 (Meier and Blobel, 1992), PKA (Chiu et al., 2002) and cdc2 kinase (Pai et al., 1995) were able to phosphorylate hNopp140, we examined the effect of doxorubicin on the phosphorylation of hNopp 140 by these protein kinases. When we examined the phosphorylation of recombinant hNopp140 by protein kinase CK2 in the presence of doxorubicin, the level of phosphorylated protein was significantly reduced as the concentration of doxorubicin increased (Fig. 3A). This reduction was not due to the inhibition of protein kinase CK2 by doxorubicin, as the phosphorylation of $\alpha$-casein by protein kinase CK2 was unaffected by doxorubicin (Fig. 3B). The effect of doxorubicin in cellular hNopp140 was further examined using the extracts of $293 \mathrm{~T}$ cells transformed with pCMV-hNopp140. When the cell extracts from $293 \mathrm{~T}$ cells or the transformed cells were subjected to 

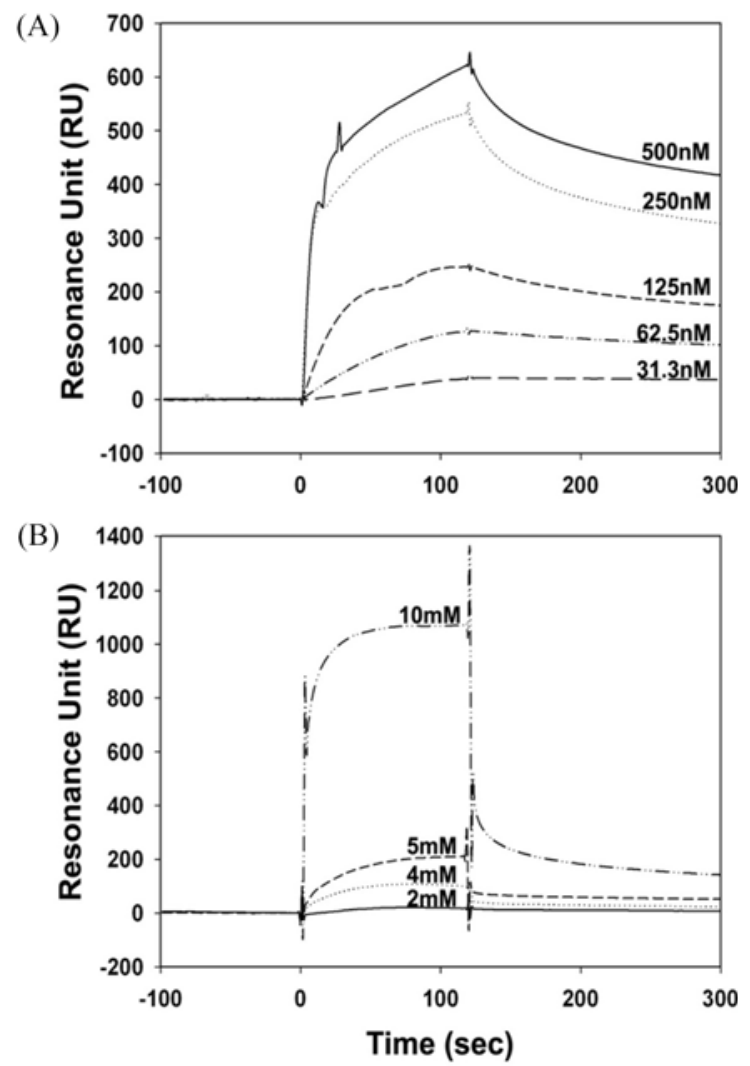

Fig. 2. The binding sensorgram of hNopp 140 on doxorubicin. (A) Binding sensorgrams were obtained with 31.3, 62.5, 125, 250 and $500 \mathrm{nM}$ of hNopp140 (from bottom to top) to a sensor chip immobilized with doxorubicin. Association and dissociation phases were started at 0 and at $120 \mathrm{~s}$, respectively. (B) Binding senorgrams of $31.3 \mathrm{nM}$ of hNopp140 were obtained in the presence of 2, 4, 5, or $10 \mathrm{mM}$ of ATP. The concentration of ATP at each measurement was indicated on top of each sensorgrams.

Western blotting using anti-hNopp140 antibodies, hNopp140 was observed in both of the extracts, and the amount of hNopp140 was much higher in the extract of transformed cells (Fig. 4, lower panel). When the extracts were reacted with protein kinase CK2 in the presence of $\left[\gamma_{-}{ }^{32} \mathrm{P}\right]$ ATP and subjected to autoradiography, a protein band at the same position of hNopp140 was detected. The phosphoryation level of this band was significantly increased in the 294T cells transformed with hNopp140 cDNA. This results was consistent to the observation that hNopp140 was a dominantly labeled protein with $\left[\gamma_{-}{ }^{32} \mathrm{P}\right]$ ATP (Meier and Blobel, 1992). It was noticeable that the apparent size both of radio-labeled protein and hNopp140 detected by Western blotting of transformed $293 \mathrm{~T}$ cells was slightly higher than the protein bands from 293 T cell. The increased size is probably due to the heavy phosphoyrlation of hNopp140 (Kim et al., 2003). These observations indicated that the dominantly radio-labeled protein from the cell extract was hNopp140. In the presence of $5 \mu \mathrm{M}$ or high concentration of doxorubicin, the viability of
(A)

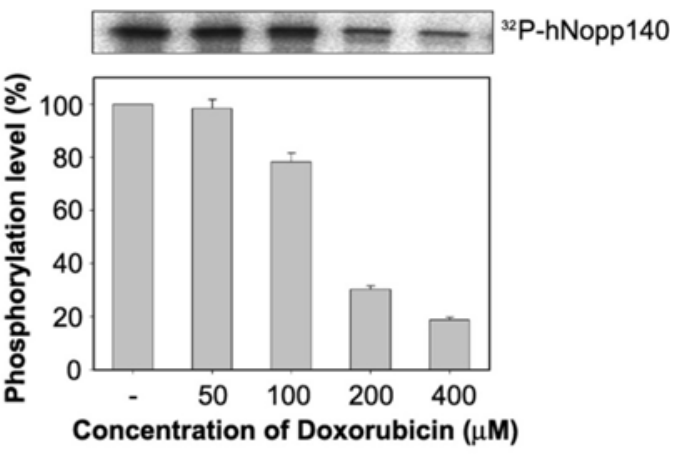

(B)

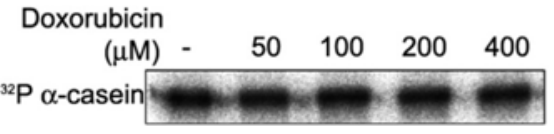

Fig. 3. Inhibition of protein kinase CK2-dependent phosphorylation of the recombinant hNopp140 by doxorubicin. (A) The effect of doxorubicin on the phosphorylation of hNopp140 was examined by isotope labeling. Recombinant hNopp 140 was incubated with protein kinase CK2 in the presence of $\left[\gamma^{-32} \mathrm{P}\right]$ ATP and increasing concentration of doxorubicin for $20 \mathrm{~min}$ at $30^{\circ} \mathrm{C}$. After the reaction, proteins were separated by SDS-PAGE, and the degree of isotope labeling on hNopp140 was measured by autoradiography. (B) The phosphorylation of $\alpha$-casein by protein kinase CK2 in the presence of $\left[\gamma^{32} \mathrm{P}\right]$ ATP and different concentration of doxorubicin was performed for $20 \mathrm{~min}$ at $30^{\circ} \mathrm{C}$. After the reaction, isotope-labeled $\alpha$-casein was detected by autoradiography.

cells was significantly reduced, and the effect of doxorubicin on the phosphorylation of hNopp140 was not able to be observed due to the toxicity of doxorubicin. The intensity of radio labeled protein was distinctly decreased in the presence of doxorubicin (Fig. 4, upper panel), indicating that doxorubicin was able to reduce the phosphorylation of hNopp140 in cell extract probably the conformation of hNopp140. These results suggest that doxorubicin may change the conformation of hNopp140 to a less accessible state to protein kinase CK2.

Phosphorylation of hNopp140 by cde2 kinase or PKA is not affected by doxorubicin. In addition to protein kinase CK2, cdc2 kinase and PKA were known to phosphorylate hNopp140. Specifically, cdc2 kinase is responsible for the elevated levels of hNopp140 phosphorylation at metaphase in cell division (Pai et al., 1995). The effect of doxorubicin on the phosphorylation of hNopp140 by cdc2 kinase and PKA was examined by measuring the amount of phosphorylation of hNopp140 in the presence of doxorubicin. Both kinases effectively phosphorylated hNopp140 (Fig. 5A and 5B upper panels). When hNopp140 reacted with cdc 2 kinase or PKA in the presence different concentrations of doxorubicin, the levels of phosphorylated hNopp140 were not changed (Figs. $5 \mathrm{~A}$ and $5 \mathrm{~B}$, lower panels). These results indicate that cdc2 kinase- or PKA-dependent phosphorylation of hNopp140 is hardly affected by doxorubicin. 


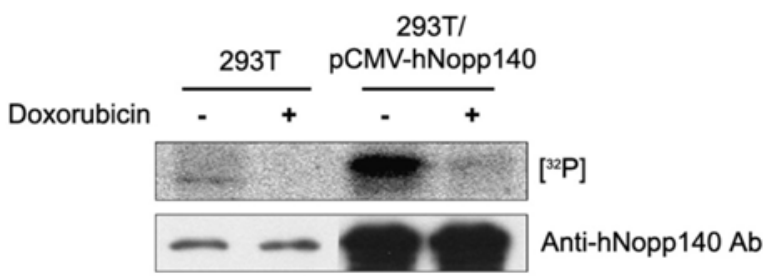

Fig. 4. Inhibition of protein kinase CK2-dependent phosphorylation of the endogenous hNopp140 by doxorubicin. Extracts from $293 \mathrm{~T}$ cells (293T) or transfected 293T cells with pCMVhNopp140 (293T-hNopp140) were incubated with protein kinase $\mathrm{CK} 2$ and $\left[\gamma^{32} \mathrm{P}\right]$ ATP in the presence or absence of doxorubicin $(100 \mu \mathrm{M})$. After the reaction, proteins in the cell extracts were separated on SDS-PAGE, and the amount of hNopp140 and the level of phosphorylation were examined by western analysis (lower panel) or autoradiography (upper panel), respectively.
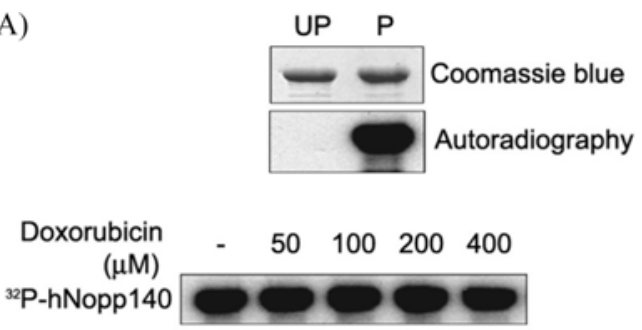

(B)
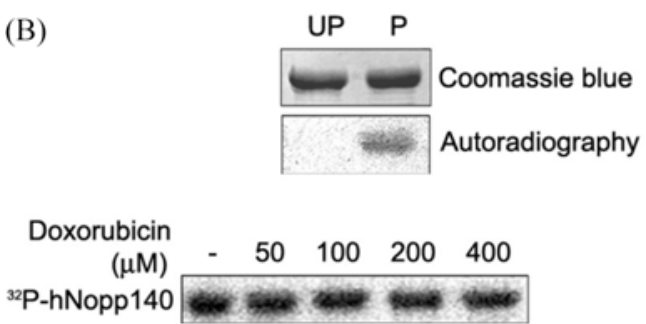

Fig. 5. Doxorubicin independent phosphorylation of hNopp 140 by cdc2 kinase and PKA kinase. hNopp140 was incubated with cdc2 kinase (A) or PKA (B) along with $\left[\gamma-{ }^{32} \mathrm{P}\right]$ ATP at 30 for 30 min. Un-phosphorylated (UP) and phosphorylated (P) hNopp140 were separated by SDS-PAGE, and examined by Coomassie staining or autoradiography (upper panel). The degree of phosphorylation of hNopp140 by the kinases in the presence of different concentrations of doxorubicin was examined by autoradiography (lower panel).

hNopp140 has characteristics of natively unfolded proteins. The sequence of hNopp140 has characteristics of natively unfolded proteins. The secondary structure predictions from the primary sequence of hNopp140 suggest that it has $>70 \%$ random coil structure (Table 1). The repetition of charged residues, as well as serine and threonine residues, might be responsible for the low percentage of secondary structure (Fink, 2005). To test whether hNopp140 has high percentage of random coil conformation or extended loop structure, the secondary structure of hNopp140 and its sensitivity against proteolysis were examined. Circular dichroism spectrometry of the recombinant hNopp140 indicated
Table 1. The percentage of secondary structures of hNopp140

\begin{tabular}{cccc}
\hline & \multicolumn{2}{c}{$\begin{array}{c}\text { Estimation from } \\
\text { sequence analysis }\end{array}$} & $\begin{array}{c}\text { Calculated from } \\
\text { CD spectrum }\end{array}$ \\
\cline { 2 - 3 } & HNN & GOR IV & \\
\hline$\alpha$-helix & 18 & 37 & 1 \\
$\beta$-strand & 0 & 0 & 33 \\
turn/random coil & 82 & 63 & 66 \\
\hline
\end{tabular}

${ }^{a}$ The secondary structure of hNopp140 was predicted from the primary sequence analysis by Hierarchical Neural Network (HNN) and GOR IV methods.

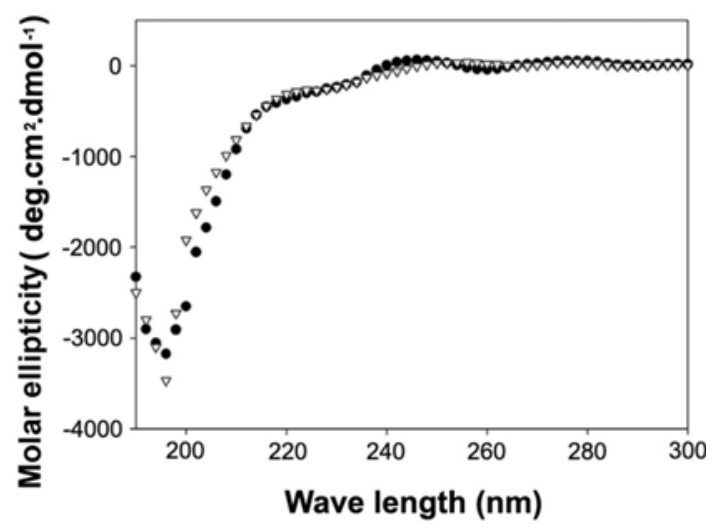

Fig. 6. CD spectra of hNopp140. The CD spectra of unphosphorylated (open triangle) or phospho-hNopp140 (filled circle) $(50 \mu \mathrm{g} / \mathrm{ml})$ were recorded in $50 \mathrm{mM}$ Tris- $\mathrm{HCl}$ buffer containing $100 \mathrm{mM} \mathrm{NaCl}(\mathrm{pH} 7.5)$ at $25^{\circ} \mathrm{C}$. The analyses were performed between 190 and $300 \mathrm{~nm}$ and data were collected for each $2 \mathrm{~nm}$ with an acquisition time of $5.0 \mathrm{~s}$.

an absorption minimum at $195 \mathrm{~nm}$ and little absorption at $220 \mathrm{~nm}$ (Fig. 6), indicating a low percentage of $\alpha$-helical or $\beta$ stranded structures (Pelton and McLean, 2000). The percentages of $\alpha$-helix, $\beta$-strand and random coil structure calculated from the spectra were $1 \%, 33 \%$ and $66 \%$, respectively. These values differ from those estimated percentages of $\alpha$-helix and $\beta$-strand from sequence analysis (Table 1). This discrepancy may be due to low ellipticity value in $210-240 \mathrm{~nm}$ range, which results in uncertainty in the calculation of secondary structure. Both un-phosphorylated or phospho-hNopp140 showed similar CD spectra, suggesting that the structural characteristics of hNopp140 are unaffected by phosphorylation. High content of random coil structure is a characteristic of intrinsically disordered proteins that are expected to be unfolded in solution, strongly resistance to heat and highly sensitive to proteolysis. The high thermostability of hNopp140 was previously demonstrated (Pai et al., 1995; Kim et al., 2003). When the sensitivity of hNopp140 to proteolysis was examined, it was degraded by subtilisin much easily than bovine serum albumin (BSA) or glutathione $\mathrm{S}$ transferase (GST) (Fig. 7A). Similarly, the degradation of hNopp 140 by protease K was much faster than those of BSA 
(A)

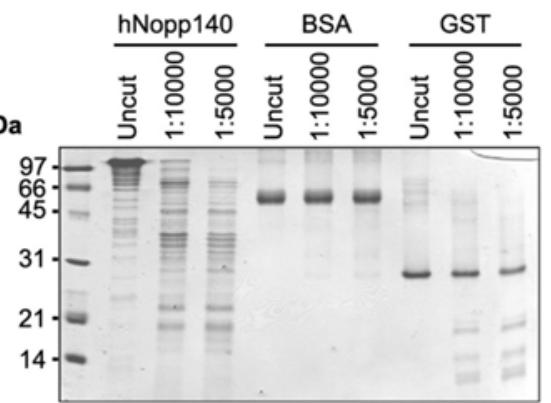

(B)

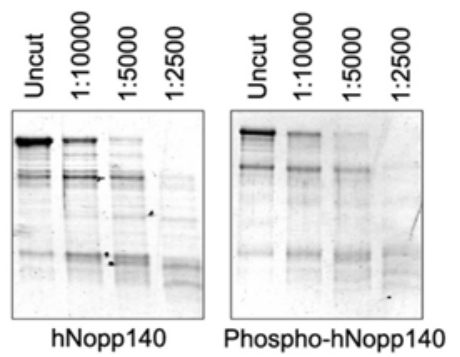

(C)

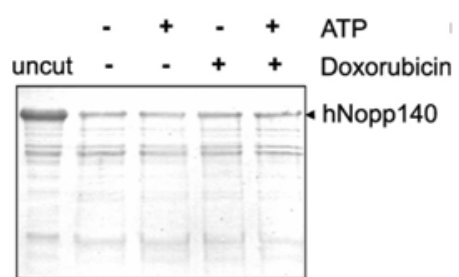

Fig. 7. Thermostability and protease-sensitivity of hNopp 140. (A) hNopp 140, BSA and GST at $0.1 \mathrm{mg} / \mathrm{ml}$ were digested at $25^{\circ} \mathrm{C}$ for $30 \mathrm{~min}$ by subtilisin at different ratios $(\mathrm{w} / \mathrm{w})$ of protease to substrate proteins. After the reaction, the proteins were separated by SDS-PAGE and visualized by Coomassie blue staining. (B) Unphosphorylated or phospho-hNopp140 were digested by subtilisin as indicated ratios, and the digested proteins were analyzed by SDS-PAGE. (C) Proteolysis of hNopp140 by subtilisin $(1: 10,000)$ was performed in the presence of ATP and/ or doxorubicin, and the digested proteins were analyzed by SDSPAGE.

or GST (data not shown). Phospho-hNopp140 was also easily degraded by subtilisin as un-phosphorylated form (Fig. 7B). Likewise, the sensitivity of hNopp140 to proteolysis was not affected by the presence of doxorubicin or ATP (Fig. 7C). These results suggest that hNopp140 has characteristics of putative natively unfolded proteins, and phosphorylation or doxorubicin hardly affects its stability against proteolysis.

\section{Discussion}

Although the involvement of hNopp140 in the formation of the nucleolus had been suggested, its cellular function is not completely understood. Detection of a highly phosphoryated hNopp140 (Pai et al., 1995) and varied levels of phosphorylation during cell cycle (Pai et al., 1996) propose that the cellular function of hNopp140 may be modulated by the degree of phosphorylation. Protein kinase CK2, cdc2 kinase and PKA were able to phosphorylate hNopp140. Specially, protein kinase CK2, which phosphorylates proteins involved in signal transduction or transcriptional control (Meggio and Pinna, 2003; Kubinski et al., 2006), was responsible for the extensive phosphorylation of hNopp140 (Pai et al., 1995). Previously, we reported that doxorubicin had a binding affinity to the Cterminus of hNopp140 (Jin et al., 2002). In this study, we further demonstrated that doxorubicin was able to mitigate the protein kinase CK2-dependent phosphorylation of hNopp140. The reduced phosphorylation of hNopp140 by doxorubicin is due to the direct interaction of doxorubicin with hNopp 140 rather than to the inhibition against the catalytic activity of protein kinase $\mathrm{CK} 2$. The doxorubicin-binding region of hNopp140 contains a number of targeting sites for protein kinase CK2, and the binding of doxorubicin to hNopp140 may induce a conformational change in which the targeting sites are no longer accessible to protein kinase CK2. When the proteolysis patterns of hNopp140 in the presence or absence of doxorubicin were examined, any significant difference was not observed (Fig. 7C), indicating that the binding of doxorubicin to hNopp140 might not induce any significant change in its folding state. It should be noted that the ability of hNopp140 to bind doxorubicin depends on its phosphorylation state: only the un-phosphorylated form exhibits high binding affinity. These results indicate that the phosphorylation at or near the doxorubicin-binding region of hNopp140 significantly decreases its binding affinity to doxorubicin, and the binding property of the C-terminus region of hNopp 140 to doxorubicin and the protein kinase CK2-dependent phosphorylation in this region are mutually exclusive. In cell, the phosphoyrlation level of hNopp140 is increased during mitosis (Pai et al., 1996). Doxorubicin may bind to hNopp140 during pre-mitotic stage and prevent further phosphoylation by CK2. Many proteins were regulated their biological function by phosphorylation. Extensive phosphorylation of hNopp140 and the fluctuation of phosphorylation level of hNopp140 during mitosis indicated that the cellular function of hNopp140 was controlled by phosphorylation. It was shown that hNopp 140 could interact with variety of cellular proteins such as RNA polymerase 1 (Yang et al., 2000), protein kinases (Li et al., 1997) or proteins constituting nucleolus (Meier and Blobel, 1994; Isaac et al., 1998). Since phosphorylation markedly alters the distribution of surface charge of proteins, the degree of phosphorylation of hNop1p40_would affect the interaction mode of hNopp140 to other proteins. Although the detailed molecular function of hNop140 has not yet been clearly uncovered, the reduced phosphorylation level of hNopp140 by doxorubicin may disrupt the cellular function of hNopp 140 by the perturbation of the interaction between hNopp140 and its binding partners.

The folding properties of hNopp140 are comparable to those of disordered protein (Fink, 2005). Although hNopp140 was shown to have thermostability (Pai et al., 1995; Kim et 
al., 2003), it showed the high sensitivity to proteases and a low percentage of secondary structures (Fig. 6, 7). It appears at odds since most thermostable proteins exhibit a strong resistance to proteolysis, high contents of stable secondary structures (Sargent et al., 1988), and low percentage flexible regions or long loop structure (Miyazono et al., 2005). Most of proteins need well-defined folded structure for the function (Lee and Yu, 2005). Recently, however, natively disordered proteins have been identified and implicated in critical eukaryotic regulatory functions such as cell cycle control, transcription and translation (Nakayama et al., 2001; Iakoucheva et al., 2002). Thermostability and high content of random coil structure are the characteristics of disordered proteins such as Tau (Schweers et al., 1994), PKI (Thomas et al., 1991), caldesmon (Bretscher, 1984; Lynch et al., 1987) and NACP (Weinreb et al., 1996). The disordered proteins distinctively have a reduced number of hydrophobic residues (I, L, V, W, F, $\mathrm{Y}, \mathrm{C}$ and $\mathrm{N}$ ) and a high percentage of hydrophilic residues (E, K, R, G, S, Q, and P) (Romero et al., 2001). The percentages of lysine and serine of hNopp140 are calculated as 15.7 and $16.7 \%$, respectively, whereas the total percentage of hydrophobic residues (I, L, V, W, F, and Y) is only $12 \%$. The unusually high ratio of charged and hydrophilic residues and the low percentage of hydrophobic hydrophilic residues also imply that hNopp140 is a putative disordered protein.

The phosphorylation-dependent interaction between hNopp140 and doxorubicin indicated that hNopp140 is an unusual disordered protein, in that it may undergo conformational change upon phosphorylation. Further analysis will confirm whether hNopp140 undergoes phosphorylation-dependent structural change, and investigation into the modulation of binding affinities to potential cellular partners, including doxorubicin, should reveal more about the cellular functions of this protein.

Acknowledgments This work was supported by grants from the 21C Frontier Research Program, Functional Proteomics Research, Ministry of Science and Technology, Korea (FPR05B2-040).

\section{References}

Blum, R. H. and Carter, S. K. (1974) Adriamycin. A new anticancer drug with significant clinical activity. Ann. Intern. Med. 80, 249-259.

Bretscher, A. (1984) Smooth muscle caldesmon. Rapid purification and F-actin cross-linking properties. J. Biol. Chem. 259, 1287312880.

Chen, H. K., Pai, C. Y., Huang, J. Y. and Yeh, N. H. (1999) Human Nopp140, which interacts with RNA polymerase I: implications for rRNA gene transcription and nucleolar structural organization. Mol. Cell. Biol. 19, 8536-8546.

Chen, H. K. and Yeh, N. H. (1997). The nucleolar phosphoprotein P130 is a GTPase/ATPase with intrinsic property to form large complexs triggered by $\mathrm{F}^{-}$and $\mathrm{Mg}^{2+}$. Biochem. Biophys. Res. Comm. 230, 370-375.
Chiu, C. M., Tsay, Y. G., Chang, C. J. and Lee, S. C. (2002) Nopp140 is a mediator of the protein kinase A signaling pathway that activates the acute phase response alpha1-acid glycoprotein gene. J. Biol. Chem. 277, 39102-39111.

Fink, A. L. (2005) Natively unfolded proteins. Curr. Opin. Struct. Biol. 15, 35-41.

Iakoucheva, L. M., Brown, C. J., Lawson, J. D., Obradovic, Z. and Dunker, A. K. (2002) Intrinsic disorder in cell-signaling and cancer-associated proteins. J. Mol. Biol. 323, 573-584.

Isaac, C., Pollard, J. W. and Meier, U. T. (2001) Intranuclear endoplasmic reticulum induced by Nopp140 mimics the nucleolar channel system of human endometrium. J. Cell Sci. 114, 4253-4264.

Isaac, C., Yang, Y. and Meier, U. T. (1998) Nopp140 functions as a molecular link between the nucleolus and the coiled bodies. J. Cell. Biol. 142, 319-329.

Jin, Y., Yu, J. and Yu, Y. G. (2002) Identification of hNopp140 as a binding partner for doxorubicin with a phage display cloning method. Chem. Biol. 9, 157-162.

Kim, Y. K., Jin, Y., Vukoti, K. M., Park, J. K., Kim, E. E., Lee, K. J. and Yu, Y. G. (2003) Purification and characterization of human nucleolar phosphoprotein 140 expressed in Escherichia coli. Protein Expr. Purif. 31, 260-264.

Koop, A. and Cobbold, P. H. (1993) Continuous bioluminescent monitoring of cytoplasmic ATP in single isolated rat hepatocytes during metabolic poisoning. Biochem. J. 295, 165170.

Kubinski, K., Zielinski, R., Hellan, U., Mazur, E. and Szyszka, R. (2006) Yeast Elf1 factor is phosphorylated and interacts with protein kinase CK2. J. Biochem. Mol. Biol. 39, 311-318.

Lee, C. and Yu, M.-H. (2005) Protein folding and diseases. J. Biochem. Mol. Biol. 38, 275-280.

Li, D., Meier, U. T., Dobrowolska, G. and Krebs, E. G. (1997) Specific interaction between casein kinase 2 and the nucleolar protein Nopp140. J. Biol. Chem. 272, 3773-3779.

Lynch, W. P., Riseman, V. M. and Bretscher, A. (1987) Smooth muscle caldesmon is an extended flexible monomeric protein in solution that can readily undergo reversible intra- and intermolecular sulfhydryl cross-linking. A mechanism for caldesmon's F-actin bundling activity. J. Biol. Chem. 262, 7429-7437.

Meggio, R. and Pinna, L. A. (2003) One-thousand-and-one substrates of protein kinase CK2. FASEB J. 17, 349-368.

Meier, U. T. and Blobel, G. (1994) NAP57, a Mammalian Nucleolar protein with a Putative Homolog in Yeast and Bacteria. J. Cell. Biol. 127, 1505-1514.

Meier, U. T. and Blobel, G. (1992) Nopp140 shuttles on tracks between nucleolus and cytoplasm. Cell 70, 127-138.

Miyazono, K., Sawano, Y. and Tanokura, M. (2005) Crystal structure and structural stability of acylphosphatase from hyperthermophilic archaeon Pyrococcus horikoshii OT3. Proteins 61, 196-205.

Nakayama, K. I., Hatakeyama, S. and Nakayama, K. (2001) Regulation of the cell cycle at the G1-S transition by proteolysis of cyclin E and p27Kip1. Biochem. Biophys. Res. Commun. 282, 853-860.

Olson, M. O. and Busch, H. (1978) Nucleolar proteins. Methods. Cell. Biol. 17, 163-210.

Pai, C. Y., Chen, H. K., Sheu, H. L. and Yeh, N. H. (1995) Cellcycle-dependent alterations of a highly phosphorylated 
nucleolar protein p130 are associated with nucleologenesis. $J$. Cell. Sci. 108, 1911-1920.

Pai, C. Y. and Yeh, N. H. (1996) Cell proliferation-dependent expression of two isoforms of the nuclear phosphoprotein p130. Biochm. Biophys. Res. Comm. 221, 581-587.

Pelton, J. T. and McLean, L. R. (2000) Spectroscopic methods for analysis of protein secondary structure. Anal. Biochem. 277, 167-176.

Romero, P., Obradovic, Z., Li, X., Garner, E. C., Brown, C. J. and Dunker, A. K. (2001) Sequence complexity of disordered protein. Proteins 42, 38-48.

Sargent, D., Benevides, J. M., Yu, M. H., King, J. and Thomas, G. J., Jr. (1988) Secondary structure and thermostability of the phage P22 tailspike: XX. Analysis by Raman spectroscopy of the wild-type protein and a temperature-sensitive folding mutant. J. Mol. Biol. 199, 491-502.

Schweers, O., Schonbrunn-Hanebeck, E., Marx, A. and Mandelkow, E. (1994) Structural studies of tau protein and Alzheimer paired helical filaments show no evidence for beta- structure. J. Biol. Chem. 269, 24290-24297.

Simpkins, H., Pearlman, L. F., and Thompson, L. M. (1984) Effects of adriamycin on supercoiled DNA and calf thymus nucleosomes studied with fluorescent probes. Cancer. Res. 44, 613-618.

Thomas, J., Van Patten, S. M., Howard, P., Day, K. H., Mitchell, R. D., Sosnick, T., Trewhella, J., Walsh, D. A. and Maurer, R. A. (1991) Expression in Escherichia coli and characterization of the heat-stable inhibitor of the cAMP-dependent protein kinase. J. Biol. Chem. 266, 10906-10911.

Weinreb, P. H., Zhen, W., Poon, A. W., Conway, K. A. and Lansbury, P. T., Jr. (1996) NACP, a protein implicated in Alzheimer's disease and learning, is natively unfolded. Biochemistry 35, 13709-13715.

Yang, Y., Isaac, C., Wang, C., Dragon, F., Pogacic, V. and Meier, U. T. (2000) Conserved composition of mammalian box $\mathrm{H} /$ ACA and box C/D small nucleolar ribonucleoprotein particles and their interaction with the common factor Nopp140. Mol. Biol. Cell. 11, 567-577. 